\title{
Method for 3D Image Representation with Reducing the Number of Frames based on Characteristics of Human Eyes
}

\author{
Kohei Arai ${ }^{1}$ \\ Graduate School of Science and Engineering \\ Saga University \\ Saga City, Japan
}

\begin{abstract}
Method for 3D image representation with reducing the number of frames based on characteristics of human eyes is proposed together with representation of $3 D$ depth by changing the pixel transparency. Through experiments, it is found that the proposed method allows reduction of the number of frames by the factor of $1 / 6$. Also, it can represent the $3 D$ depth through visual perceptions. Thus, real time volume rendering can be done with the proposed method.
\end{abstract}

Keywords-3D image representation; Volume rendering; NTSC image display

\section{INTRODUCTION}

Computer input by human eyes only is proposed and implemented [1]-[3] together with its application to many fields, communication aids, electric wheel chair controls, having meal aids, information collection aids (phoning, search engine, watching TV, listening to radio, e-book/e-comic/elearning/,etc., domestic helper robotics and so on [4]-[15]. In particular, the proposed computer input system by human eyes only does work like keyboard as well as mouse. Therefore, not only key-in operations but also mouse operations (right and left button click, drag and drop, single and double click) are available for the proposed system.

It is well known that hands, fingers operation is much slower than line of sight vector movements. It is also known that accidental blink is done within 0.3 second. Therefore, the proposed computer input system by human eyes only decides the specified key or location when the line of vector is fixed at the certain position of computer display for more than 0.3 second. In other words, the system can update the key or the location every 0.3 second. It is fast enough for most of all application fields. Meanwhile, 3D image display and manipulation can be done with 3D display. Attempts are also done with 2D display for 3D image display and manipulation [16],[17], on the other hands. Most of previous attempts are based on touch panel based manipulation by hands and fingers. As aforementioned, eyes operations are much faster than hands \& fingers operations. Therefore, 3D image display and manipulation method by human eyes only is proposed in this paper.

3D image representations are widely used for a variety of applications such as medical electronics diagnostics image display, LSI pattern designs, and so on. Volume rendering is most popular method for 3D image representations, in general. It, however, takes a huge computational resources.

Volume rendering is in general costly. Real time representation, therefore, is not easy for volume rendering even for grid computing is used. For instance, 6 PCs of grid computing can be reduced by $35 \%$ of process time for the 3D representation with volume rendering. In order to reduce the process time of volume rendering, the number of frames which have to be display is reduced by using afterimage phenomenon. Time resolution of human eyes ranges from 50 to $100 \mathrm{~ms}$. Illumination switching between on and off within the 50 to $100 \mathrm{~ms}$ is recognized as continuous illumination by human eyes. Therefore, it can be done to reduce the number of frames for 3D object image representation by using multilayer representation. On the other hand, refresh cycle of the NTSC video signal corresponds to $33 \mathrm{~ms}$. Therefore, the interval of refresh cycle has to be within $33 \mathrm{~ms}$ for the proposed volume rendering.

The next section describes the proposed system followed by experiment. Then concluding remarks are described with some discussions.

\section{PROPOSED METHOD}

\section{A. Basic Idea}

Basic Idea for the Proposed Method 3D model that displays a picture or item in a form that appears to be physically present with a designated structure. Essentially, it allows items that appeared flat to the human eye to be display in a form that allows for various dimensions to be represented. These dimensions include width, depth, and height. 3D model can be displayed as a two-dimensional image through a process called 3D rendering or used in a computer simulation of physical phenomena. The model can also be physically created using 3D printing devices. Models may be created automatically or manually. The manual modeling process of preparing geometric data for 3D computer graphics is similar to plastic arts such as sculpting.

There are some previously proposed methods for 3D display such as tracing object contour and reconstruct 3D object with line drawings, and wireframe representation and display 3D object with volume rendering. There is another method, so called OCT: Optical Coherence Tomography. It, 
however, quit expensive than the others. Fundamental idea of the proposed method is afterimage. Response time of human eyes is that the time resolution of human eyes: $50 \mathrm{~ms} \sim 100 \mathrm{~ms}$. An afterimage or ghost image or image burn-in is an optical illusion that refers to an image continuing to appear in one's vision after the exposure to the original image has ceased. One of the most common afterimages is the bright glow that seems to float before one's eyes after looking into a light source for a few seconds. Afterimages come in two forms, negative (inverted) and positive (retaining original color). The process behind positive afterimages are unknown, though thought to be related to neural adaptation. On the other hand, negative afterimages are a retinal phenomenon and are well understood. Example of 3D image on to 2D display is shown in Fig.1. This is the proposed system concept. In the example, "A" marked 3D image of multi-fidus which is acquired with CT scanner is displayed onto computer screen. "B", "C", ... are behind it.

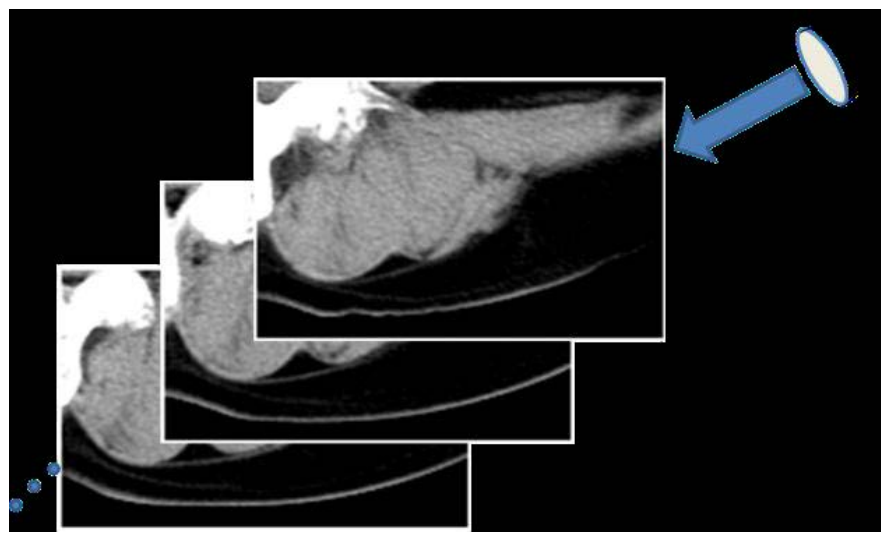

Fig. 1. System Concept

Such this layered 3D images are aligned with depth direction. Attached character "A" to "Z" are transparent and displayed at just beside the layered image at the different locations. Therefore, user can recognize the character and can select one of those characters by their eye. Arrow shows the line of sight vector. Curser can be controlled by human eyes only. By sweeping the character, 3D images are displayed by layer by layer. Therefore, it looks like time division delay of 3D images.

\section{B. Displayed Image in Automatic Mode}

Implementation of the proposed system is conducted. By using mouse operation by human eyes only, 3D image with different aspects can be recreated and display. It is confirmed that conventional image processing and analysis can be done with mouse operation by human eyes only.

Fig. 2 shows the example of displayed layered images. In this example, 1024 of layer images are prepared for 3D object. By displaying the prepared layered images alternatively in automatic mode, 3D object appears on the screen. Furthermore, as shown in Fig.2, internal structure is visible other than the surface of the 3D object. It looks like a semitransparent 3D surface and internal structure in side of the 3D objects.
Another example is shown in Fig.3. In the figure, the first to $768^{\text {th }}$ layer images are shown together with a slant view of the 3D object image with the several layer images. 1024 layer of images are created for the 3D object by using Open GL. Surface rendering with the 1024 layer of images makes a 3D object rendering like Fig.3.

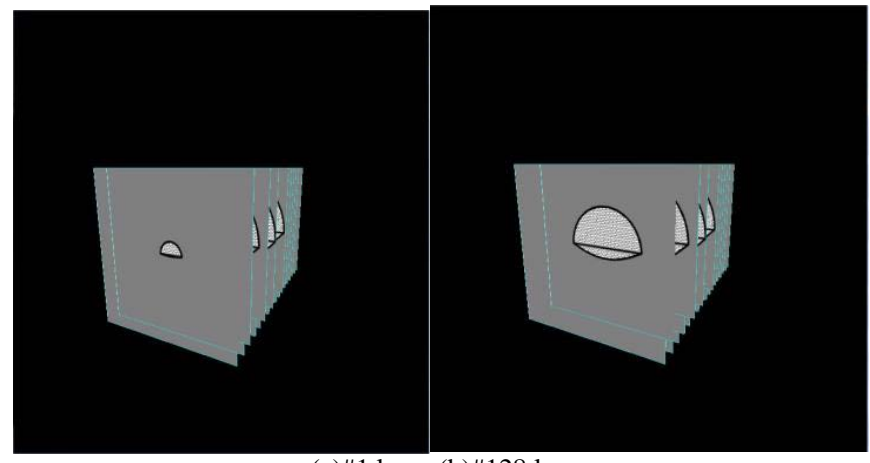

(a)\#1 layer (b)\#128 layer

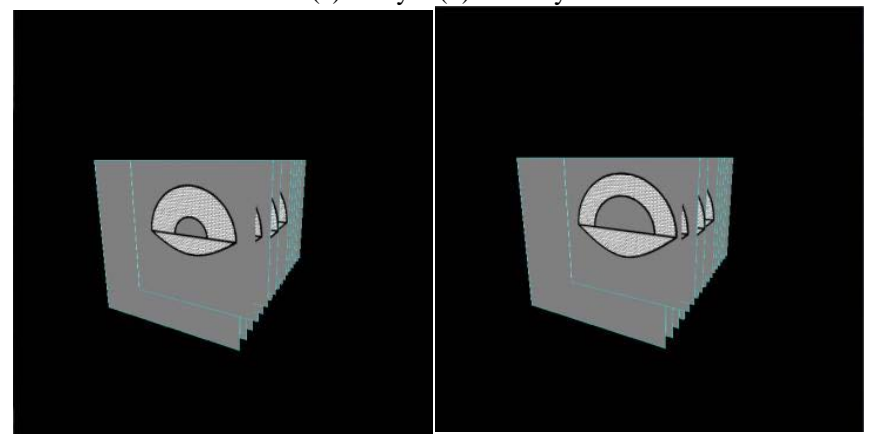

(c)\#256 layer (d)\#512 layer

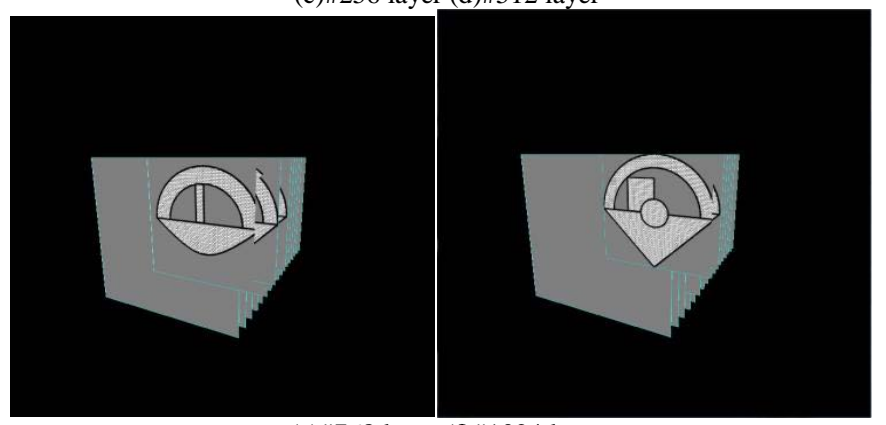

(e)\#768 layer (f)\#1024 layer

Fig. 2. Example of displayed layered images

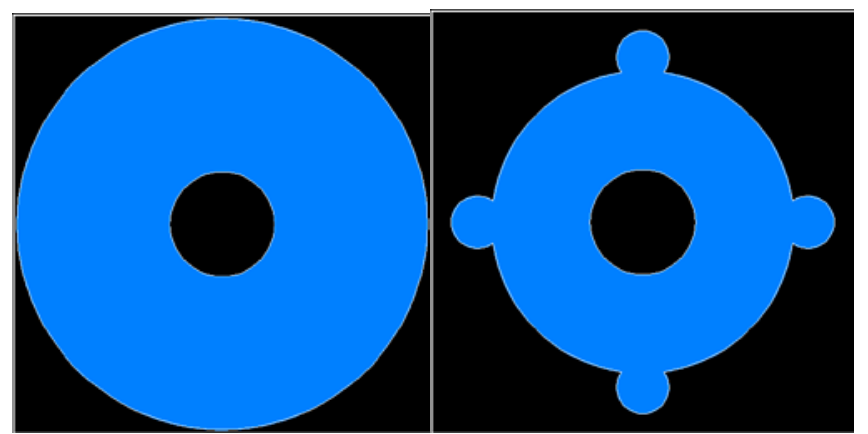

(a)\#1 layer (b)\#128 layer 


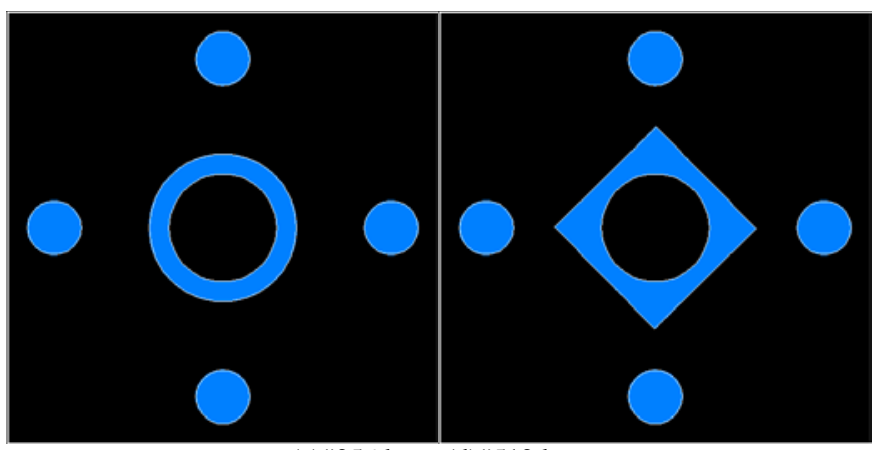

(c)\#256 layer (d)\#512 layer

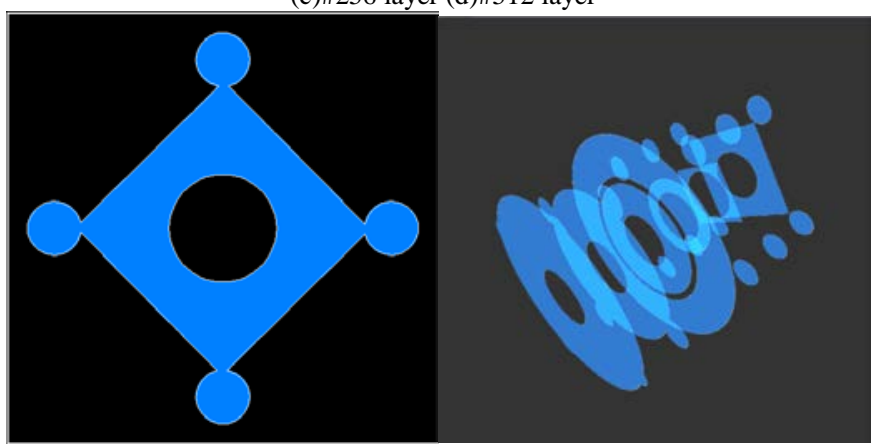

(e)\#768 layer (f)several layer representation

Fig. 3. Example of 3D object image representation

Fig.4 shows the examples of 3D object representation images created with the 1024 layer of images which are shown in Fig. 3 from the different aspects. There are ambiguities for the depth representation in the images of Fig.4. This is because of hidden line and surface distinguish process based on depth buffer method. Therefore, resultant image of the layer images surface rendering cannot represent 3D object properly which is shown in Fig.5.

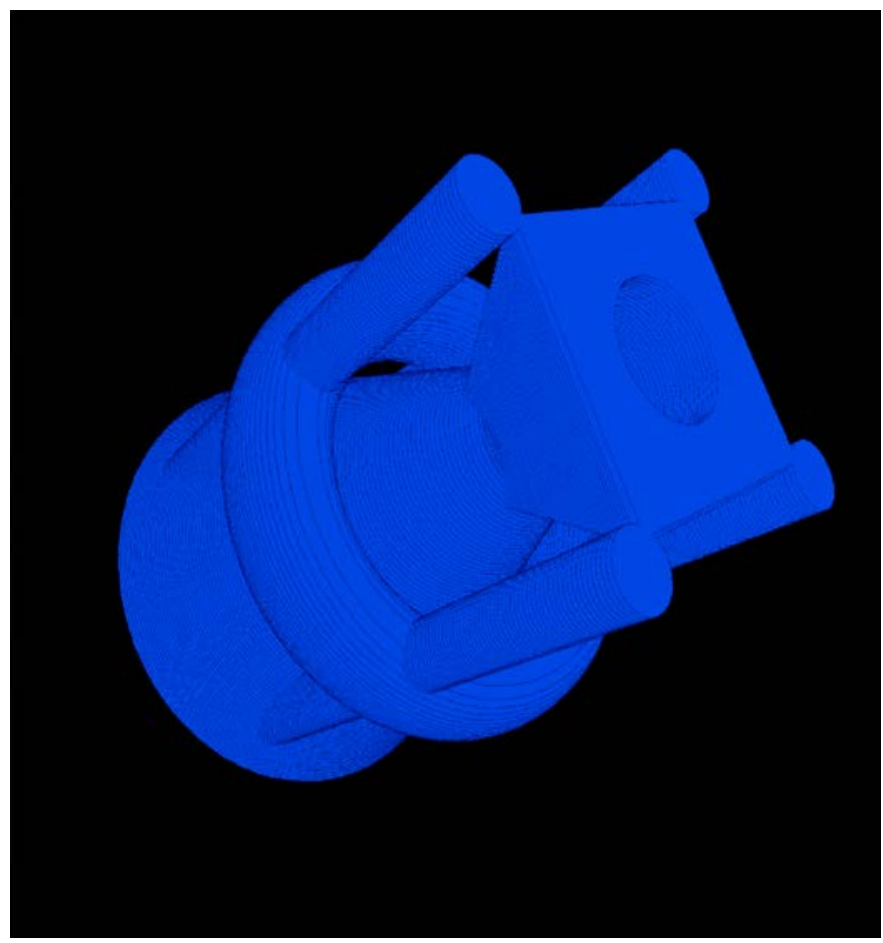

(a)3D object image representation (side view from the right)

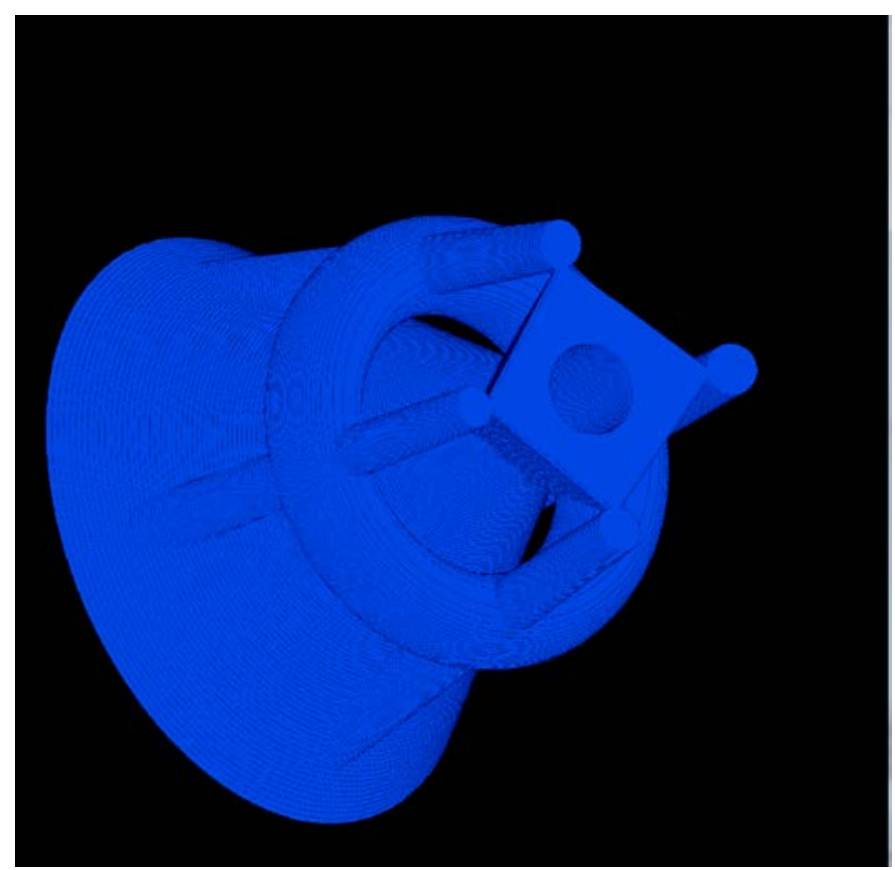

(b)3D object image representation (side view from the left)

Fig. 4. 3D object image representation from the different aspects

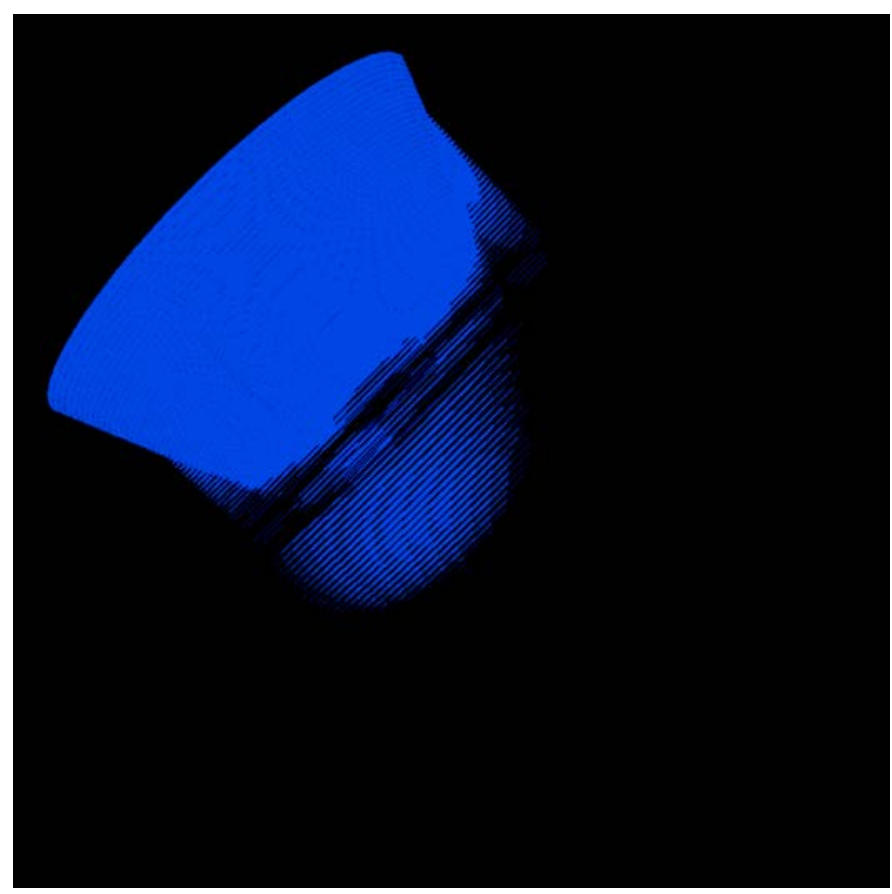

Fig. 5. 3D object image representation with hidden line and surface distinguish

\section{Changing the Transparency}

In order to represent the depth information, transparency is used. The purpose of the depth information representation is to express the internal structure of the $3 \mathrm{D}$ object. By changing the transparency, somewhat depth information can be represented as shown in Fig.6. In the figure, 100\% denotes 0\% transparent image while 5\% also denotes 95\% transparent image, respectively. It is clear that internal structure can be seen by changing transparency. Therefore, users can select the 
transparency depending on which portion of internal structure would like to see through visual perception.

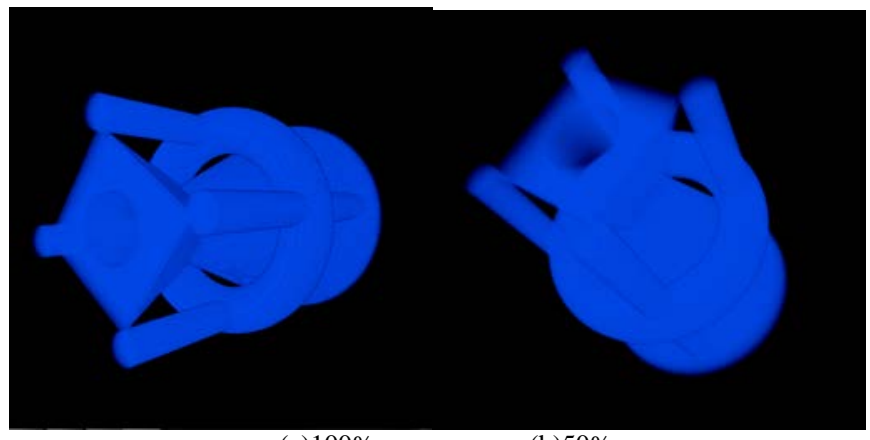

(a) $100 \%$

(b) $50 \%$

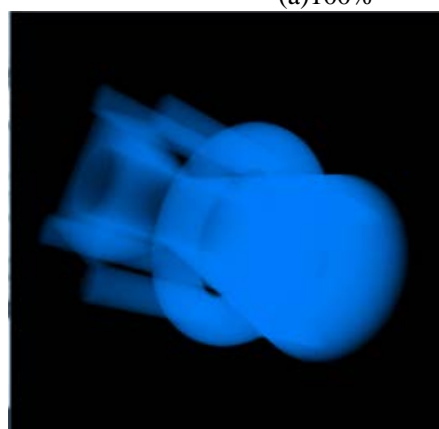

(c) $10 \%$

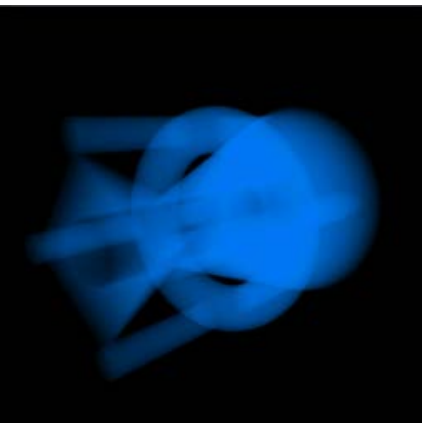

(d) $5 \%$

Fig. 6. Examples of 3D object image representation with changing transparency

\section{Foggy Representation for Depth Representations}

In order to represent the depth information, not only transparency but also foggy representation is added. Because of depth information representation is getting week depending on the transparency, it is needed to add some other representation for depth information. One of the effect is foggy representation with the different color.

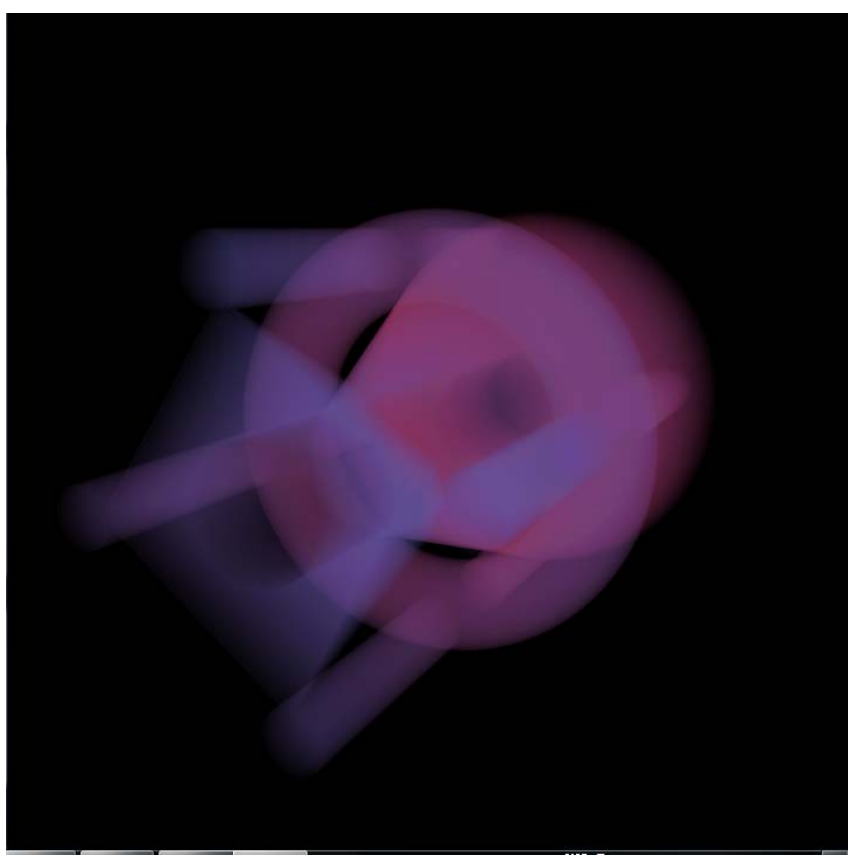

(a)Fog added 3D image for depth representation

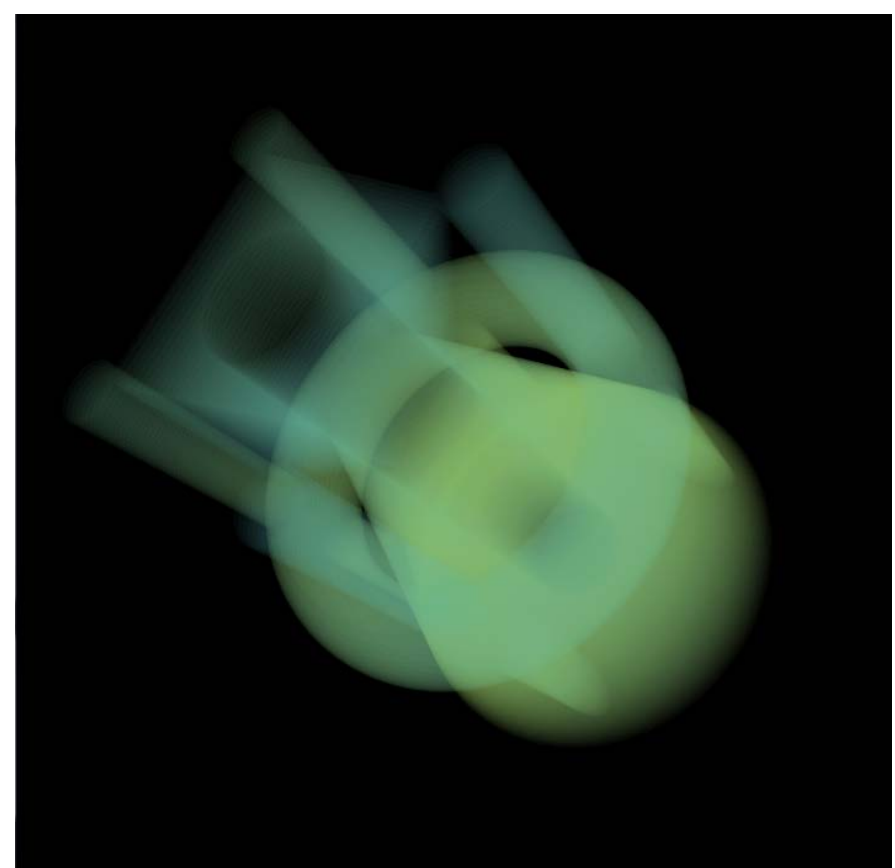

(b) Fog added 3D image for depth representation

Fig. 7. Examples of Fog added 3D image for depth representation

Fig.7 shows examples of foggy representation of depth information representations.

\section{E. Implementation}

Implementation is done based on Open GL. Parameter setting can be done with the slide ruler shown in Fig.8.

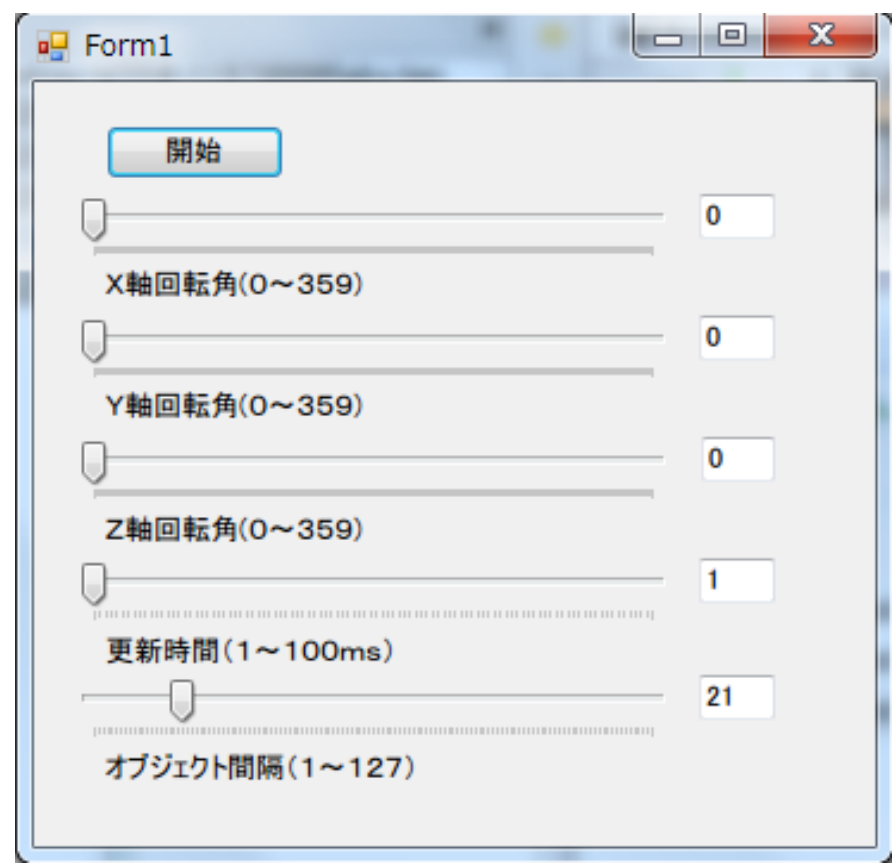

Fig. 8. Scroll slide bar for designation of parameters of 3D object image representations (the slide ruler is for the rotation angle in $\mathrm{x}$ axis followed by $\mathrm{y}$ and $\mathrm{z}$ axis and refresh cycle of the time interval of display as well as object frame interval ranges from 1 to 127

Slid rulers for rotation angles in $\mathrm{x}, \mathrm{y}$, and $\mathrm{z}$ axis which ranges from 0 to 355 degree, refresh cycle of time interval 
which ranges from 1 to $100 \mathrm{~ms}$, and object interval (the number of frames which ranges from 1 to 127) are available to set. The other parameter, such as transparency (ranges from 0 to $100 \%$ ), foggy representation can be set automatically.

As mentioned above, the time resolution of human eyes ranges from 50 to $100 \mathrm{~ms}$. Illumination switching between on and off within the 50 to $100 \mathrm{~ms}$ is recognized as continuous illumination by human eyes. Therefore, it can be done to reduce the number of frames for 3D object image representation by using multi-layer representation. On the other hand, refresh cycle of the NTSC video signal corresponds to $33 \mathrm{~ms}$. Therefore, the interval of refresh cycle has to be within $33 \mathrm{~ms}$ for the proposed volume rendering. Example of a frame image of the proposed volume rendering is shown in Fig.9.

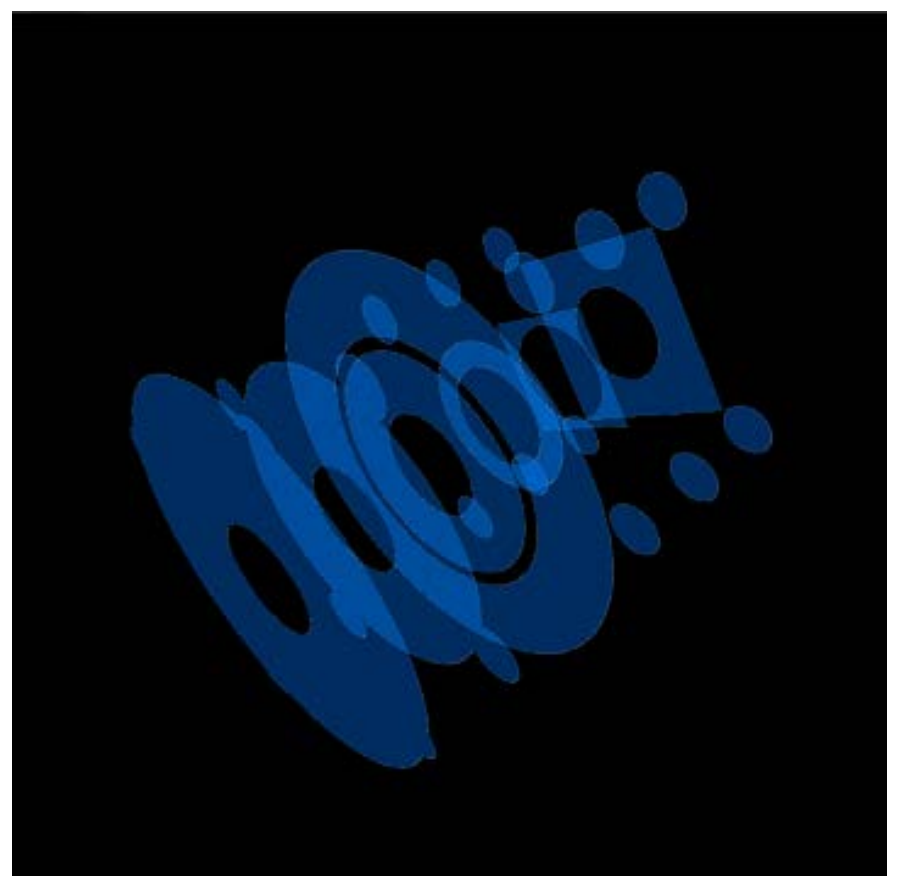

Fig. 9. Example of a frame image of the proposed volume rendering

\section{EXPERIMENT}

3D object image representation based on the proposed method with the image datasets used in this experiment were from the Laboratory of Human Anatomy and Embryology, University of Brussels (ULB), Belgium. Fig.10 shows examples of the resultant images. The $3 \mathrm{D}$ images used are derived from CT scan images. In these cases, the number of frames used is 127 while the transparency is set at $50 \%$. Meanwhile, the refresh cycle of the time interval is set at 17 ms which corresponds to $58 \mathrm{f} / \mathrm{s}$. Thus, $1 / 6$ of reduction can be achieved successfully.

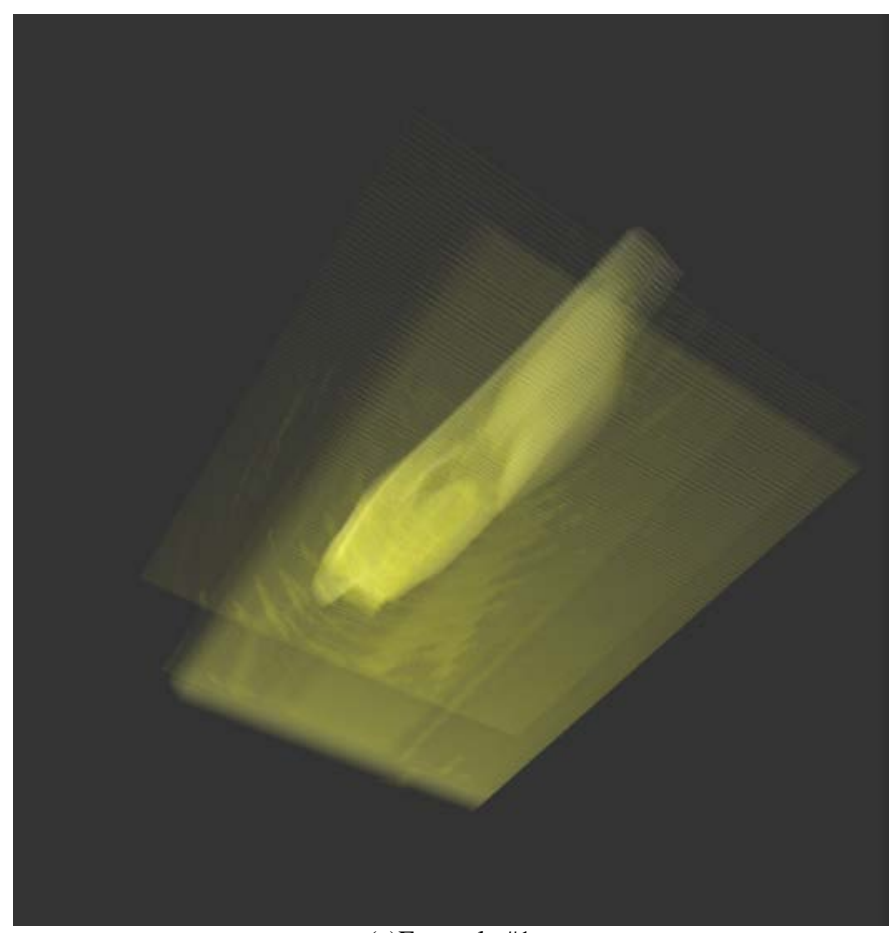

(a)Example \#1

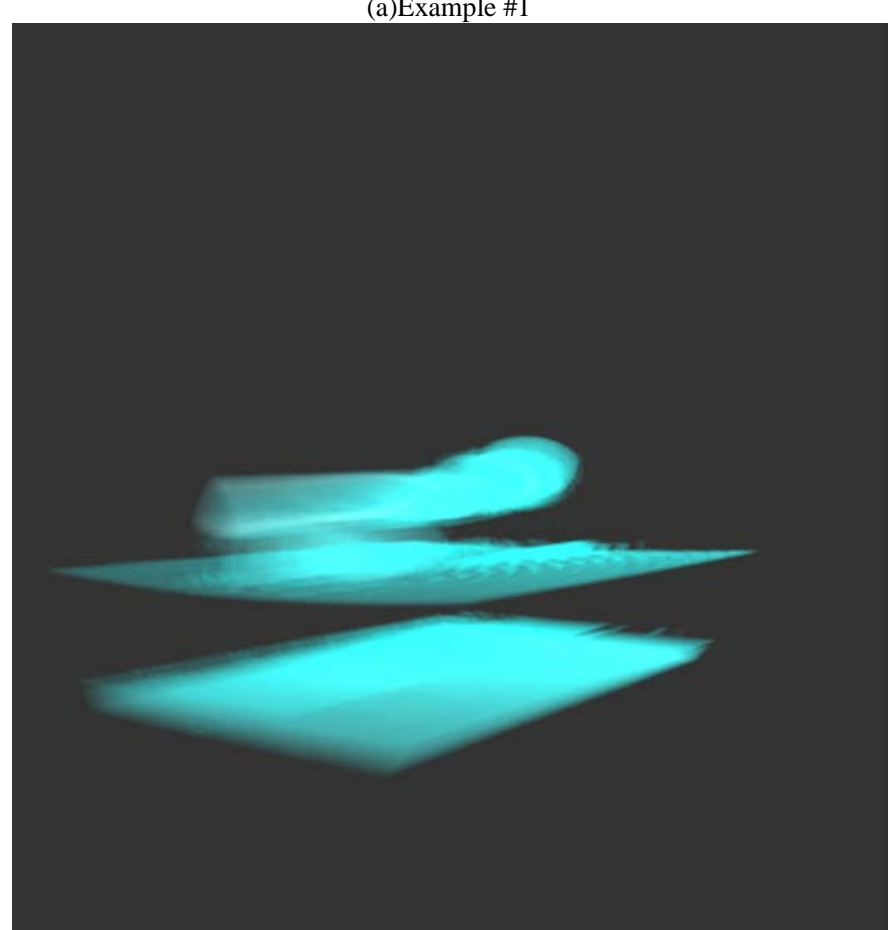

(b)Example \#2

Fig. 10. 3D object image representation based on the proposed method with the image datasets used in this experiment were from the Laboratory of Human Anatomy and Embryology, University of Brussels (ULB), Belgium 


\section{CONCLUSION}

Method for 3D image representation with reducing the number of frames based on characteristics of human eyes is proposed together with representation of 3D depth by changing the pixel transparency. Through experiments, it is found that the proposed method allows reduction of the number of frames by the factor of $1 / 6$. Also, it can represent the 3D depth through visual perceptions. Thus, real time representation of 3D object can be displayed onto computer screen.

Further investigations are required for improvement of frame reduction ratio. The refresh cycle has to be optimized. Also, the transparency and foggy representation has to be optimized. In other word, a method for optimization of the parameters has to be created.

\section{REFERENCES}

[1] K.Arai, H.Uwataki, Computer input by human eyes only based on cornea center extraction which allows users' movements, Journal of Institute of Electric Engineering of Japan, C-127, 7, 1107-1114, 2007.

[2] K.Arai, M.Yamaura, Improvement of blink detection performance based on Morphologic filter for computer input by human eyes only, Journal of Image Electronics Engineering Society of Japan, 37, 5, 601-609, 2008.

[3] K.Arai, K.Yajima, Communication aid based on computer input by human eyes only, Journal of Institute of Electric Engineering of Japan, C-128, 11, 1679-1686, 2008.

[4] D. Purwanto, R. Mardiyanto and K. Arai, Electric wheel chair control with gaze detection and eye blinking, Artificial Life and Robotics, AROB Journal, 14, 694,397-400, 2009.

[5] K.Arai, R. Mardiyanto, Computer input by human eyes only with blink detection based on Gabor filter, Journal of Visualization Society of Japan, 29,Suppl.2, 87-90,2009.

[6] K. Arai and R. Mardiyanto, Real time blinking detection based on Gabor filter, International Journal of Human Computer Interaction, 1, 3, 33-45, 2010.

[7] K. Arai and M. Yamaura, Computer input with human eyes only using two Purkinje images which works in a real time basis without calibration, International Journal of Human Computer Interaction, 1, 3, 71-82, 2010.

[8] K. Arai and R. Mardiyanto, Camera mouse and keyboard for handicap person with trouble shooting capability, recovery and complete mouse events, International Journal of Human Computer Interaction, 1, 3, 4656, 2010.

[9] K. Arai, R. Mardiyanto, A prototype of electric wheel chair control by eye only for paralyzed user, Journal of Robotics and Mechatronics, 23, 1, 66-75, 2010
[10] K. Arai, K. Yajima, Robot arm utilized having meal support system based on computer input by human eyes only, International Journal of Human-Computer Interaction, 2, 1, 120-128, 2011.

[11] Kohei Arai, Ronny Mardiyanto, Autonomous control of eye based electric wheel chair with obstacle avoidance and shortest path finding based on Dijkstra algorithm, International Journal of Advanced Computer Science and Applications, 2, 12, 19-25, 2011.

[12] K. Arai, R. Mardiyanto, Eye-based human-computer interaction allowing phoning, reading e-book/e-comic/e-learning, Internet browsing and TV information extraction, International Journal of Advanced Computer Science and Applications, 2, 12, 26-32, 2011.

[13] K. Arai, R. Mardiyanto, Eye based electric wheel chair control systemI(eye) can control EWC-, International Journal of Advanced Computer Science and Applications, 2, 12, 98-105, 2011.

[14] K. Arai, R. Mardiyanto, Evaluation of users' impact for using the proposed eye based HCI with moving and fixed keyboard by using eeg signals, International Journal of Research and Reviews on Computer Science, 2, 6, 1228-1234, 2011.

[15] K. Arai, R. Mardiyanto, Electric wheel chair controlled by human eyes only with obstacle avoidance, International Journal of Research and Reviews on Computer Science, 2, 6, 1235-1242, 2011

[16] K.Arai, X.Y.Guo, Method for 3D object of content representation and manipulations on 2D display using human eyes only, Proceedings of the International Conference on Convergence Content 2012, 49-50, 2012.

[17] Kohei Arai, Method for 3D rendering based on intersection image display which allows representation of internal structure of 3D objects, International Journal of Advanced Research in Artificial Intelligence, 2, 6, 46-50, 2013.

\section{AUTHORS PROFILE}

Kohei Arai, He received BS, MS and PhD degrees in 1972, 1974 and 1982, respectively. He was with The Institute for Industrial Science and Technology of the University of Tokyo from April 1974 to December 1978 and also was with National Space Development Agency of Japan from January, 1979 to March, 1990. During from 1985 to 1987, he was with Canada Centre for Remote Sensing as a Post Doctoral Fellow of National Science and Engineering Research Council of Canada. He moved to Saga University as a Professor in Department of Information Science on April 1990. He was a councilor for the Aeronautics and Space related to the Technology Committee of the Ministry of Science and Technology during from 1998 to 2000. He was a councilor of Saga University for 2002 and 2003. He also was an executive councilor for the Remote Sensing Society of Japan for 2003 to 2005. He is an Adjunct Professor of University of Arizona, USA since 1998. $\mathrm{He}$ also is Vice Chairman of the Commission-A of ICSU/COSPAR since 2008. He received Science and Engineering Award of the year 2014 from the minister of the ministry of Science Education of Japan and also received the Bset Paper Award of the year 2012 of IJACSA from Science and Information Organization: SAI. In 2016, he also received Vikram Sarabhai Medal of ICSU/COSPAR and also received 20 awards. He wrote 34 books and published 520 journal papers. He is Editor-in-Chief of International Journal of Advanced Computer Science and Applications as well as International Journal of Intelligent Systsems and Applications. http://teagis.ip.is.sagau.ac.jp/ 